\title{
ACUMULAÇÕES DE GÁS NOS SEDIMENTOS DA PLATAFORMA CONTINENTAL INTERNA DO RIO GRANDE DO SUL
}

\author{
Laurício Corrêa Terra, FURG; Lauro Júlio Calliari, FURG; Gilberto Henrique Griep, FURG.
}

Copyright 2014, SBGf - Sociedade Brasileira de Geofísica

Este texto foi preparado para a apresentação no VI Simpósio Brasileiro de Geofísica, Porto Alegre, 14 a 16 de outubro de 2014. Seu conteúdo foi revisado pelo Comitê Técnico do VI SimBGf, mas não necessariamente representa a opinião da SBGf ou de seus associados. É proibida a reprodução total ou parcial deste material para propósitos comerciais sem prévia autorização da SBGf.

\section{Resumo}

Feições anômalas foram registradas através de dados sísmicos de alta resolução $(3,5 \mathrm{kHz})$ na plataforma continental interna do Rio Grande do Sul (RS) e foram interpretadas como presença de gás nos sedimentos de subsuperfície. Foram utilizados cinco perfis de três linhas sísmicas adquiridas em um cruzeiro oceanográfico durante o programa Amazônia Azul. O equipamento de aquisição dos registros sísmicos foi o perfilador Chirp Sonar de subsuperfície modelo Bathy 2010 preso ao navio oceanográfico Atlântico Sul da Universidade Federal do Rio Grande. As concentrações de gás podem ter se originado a partir da degradação da matéria orgânica depositada em ambientes possivelmente lagunares.

\section{Introdução}

O estudo da subsuperfície de áreas submersas rasas tem sido de extrema importância nas últimas décadas devido às atividades humanas crescentes e cada vez mais intensa em regiões costeiras. Dentre algumas destas atividades vale a pena ressaltar a prospecção de recursos minerais.

A indústria petrolífera, por exemplo, acompanhada do crescimento tecnológico e em busca de novas jazidas, vem migrando suas prospecções da costa para o oceano (Neto \& Costa, 2007).

A aquisição de dados geofísicos em áreas marinhas através do método sísmico tem sido amplamente empregada na exploração de combustíveis fósseis (Gausland, 2003; Vilardo, 2007).

Dados geofísicos têm revelado a ocorrência de gás acumulado nos sedimentos em diversos ambientes costeiros do mundo (Garcia-Garcia et al., 1999; Manley \& Flood, 1989; Park et al., 1991; Taylor et al., 2000).

No Brasil, a ocorrência de gás em sedimentos também foi detectada por vários autores; Baía de Guanabara / RJ (Baptista Neto et al., 1996; Quaresma et al., 2000), Plataforma Continental do Amazonas (Costa \& Figueiredo, 1998), Lagoa dos Patos / RS (Weschenfelder et al., 2006; Vasconcellos et al. 2006; Vasconcellos, 2009).

Durante o programa Amazônia Azul, foi realizado pelo Laboratório de Oceanografia Geológica da Universidade Federal do Rio Grande (LOG/FURG) um cruzeiro oceanográfico utilizando sísmica rasa na plataforma continental do Rio Grande do Sul. Sedimentos quaternários revelaram perfis com anomalias acústicas que foram relacionados a ocorrências de gás.

A área de estudo está compreendida em perfis de três linhas sísmicas $(4040,0922,4551)$ localizadas na plataforma continental interna rasa do Rio Grande do Sul em frente ao município de Rio Grande, no estado brasileiro do Rio Grande do Sul (Fig. 1).

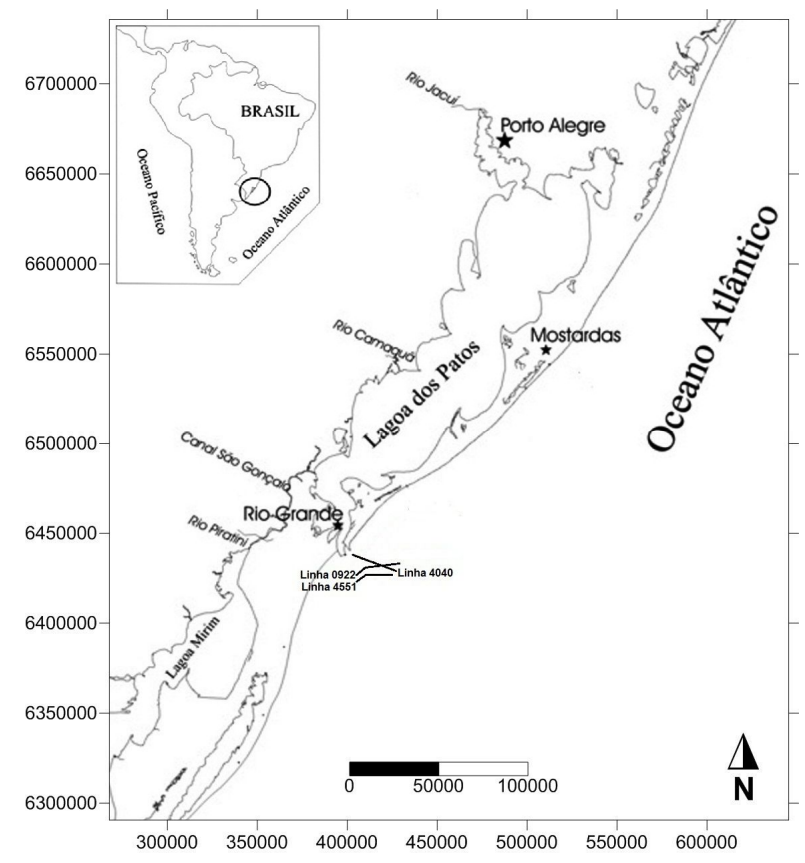

Figura 1 - Mapa de localização das linhas sísmicas.

O propósito deste estudo é caracterizar esses perfis anômalos, procurando entender o modo de formação de gás dentro do contexto geológica.

\section{Metodologia}

Os dados geofísicos foram coletados a bordo do Navio Oceanográfico Atlântico Sul pertencente à Universidade Federal do Rio Grande durante cruzeiros do programa Amazônia Azul.

O equipamento utilizado para obtenção das linhas sísmicas foi o perfilador de subsuperfície Bathy 2010 Chirp Sub Bottom Profiler, composto por um sistema de aquisição de dados (Sonar Unit) Bathy 2010, servidor Bathy 2010, transmissor de força linear 5-30KW (LPT Linear Power Transmitter), e quatro transdutores de diferentes frequências: TR-109 $3.5 \mathrm{KHz}, \mathrm{TC}-12 / 34$ $12 \mathrm{KHz}$ de banda larga (Widebeam Transducer), TC$12 \mathrm{NB} 12 \mathrm{KHz}$ banda estreita (Narrowbeam Transducer), TC-2084 33KHz. Os transdutores foram fixados na lateral do casco da embarcação, servindo simultaneamente como emissor e receptor do sinal acústico.

O tratamento das imagens sísmicas foi realizado no software SonarWiz 5.0. Este permitiu a escolha do padrão de visualização de imagem, utilização de filtros de ruídos e marcação das estruturas. 


\section{Resultados}

$\mathrm{Na}$ área de estudo foi visualizado cinco perfis (Fig. 2) com feições anômalas que apresentam turbidez nos registros. Estas feições foram interpretadas como bolsões de gás acumuladas nos sedimentos.

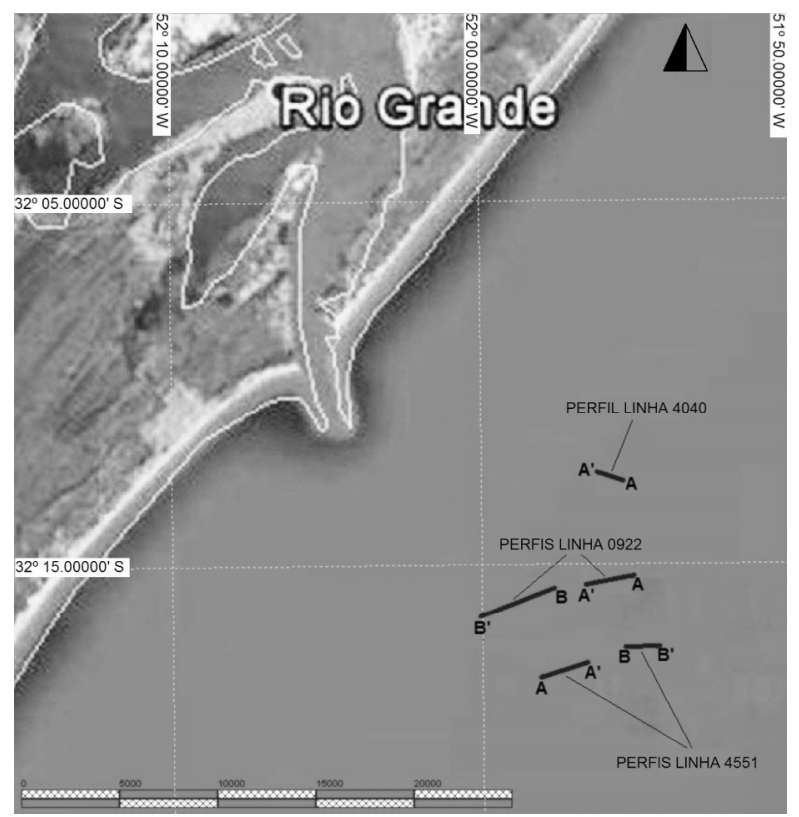

Figura 2 - Mapa de localização dos perfis sísmicos.

O perfil sísmico A - A' (Fig. 3) registrado na linha 4040 mostra uma paleosuperfície com uma cortina refletiva anômala indicando gás. Esta cortina está compreendida entra as coordenadas 32 $12,7070^{\prime}$ 'S / $51^{\circ} 55,4544^{\prime} \mathrm{W}$ e $32^{\circ} 12,4789^{\prime} \mathrm{S} / 51^{\circ}$ 56,2273'W, possui largura de $1285 \mathrm{~m}$ e está a uma profundidade de $10 \mathrm{~m}$ em relação ao fundo marinho.

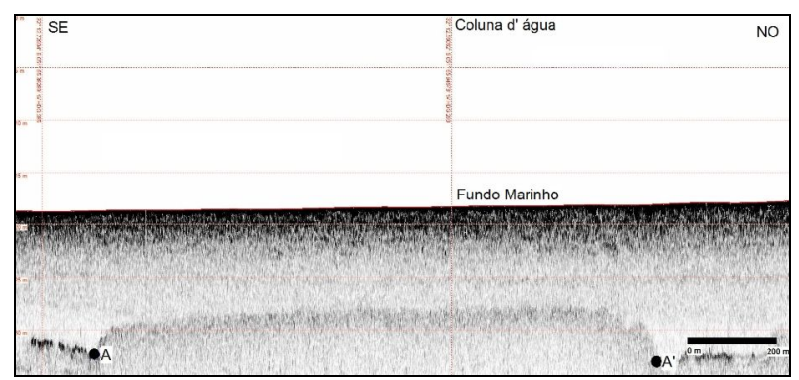

Figura 3 - Perfil sísmico de gás da linha 4040.

O perfil sísmico A - A' (Fig. 4a) registrado na linha 0922 está compreendido entra as coordenadas $32^{\circ}$ $15,3401^{\prime} \mathrm{S} / 51^{\circ} 55,0922^{\prime} \mathrm{W}$ e $32^{\circ}$ 15,5206'S / 51응 56,6031 'W, possui largura de $1.700 \mathrm{~m}$ e está a uma profundidade de $8,4 \mathrm{~m}$ em relação ao fundo marinho.

O Perfil sísmico B - B' (Fig. 4b) registrado na linha 0922 está compreendido entra as coordenadas $32^{\circ}$

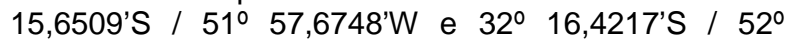
00,0436 'W, possui largura de $5.000 \mathrm{~m}$ e está a uma profundidade de $8,6 \mathrm{~m}$ em relação ao fundo marinho.

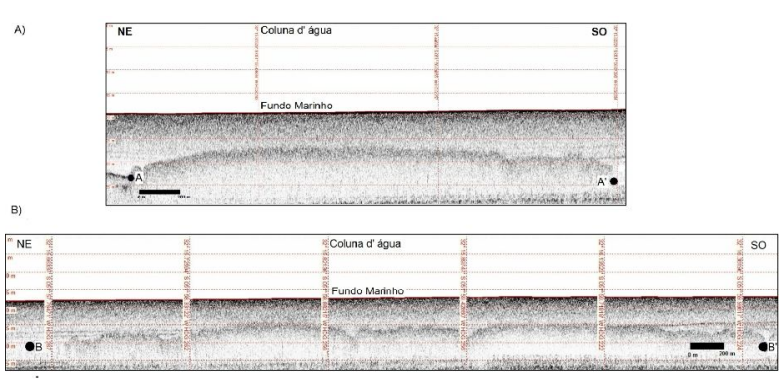

Figura 4 - Perfis sísmicos de gás da linha 0922.

O perfil sísmico $A-A^{\prime}$ (Fig. 5a) registrado na linha 4551 está compreendido entra as coordenadas $32^{\circ}$

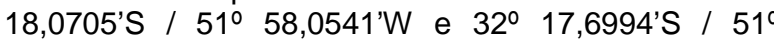
56,6152 'W, possui largura de 2.353 m e está a uma profundidade de $10,52 \mathrm{~m}$ em relação ao fundo marinho.

O Perfil sísmico B - B' (Fig. 5b) registrado na linha 4551 está compreendido entra as coordenadas $32^{\circ}$

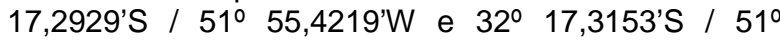
$54,3901^{\prime} \mathrm{W}$, possui largura de $1.627 \mathrm{~m}$ e está a uma profundidade de $12 \mathrm{~m}$ em relação ao fundo marinho.

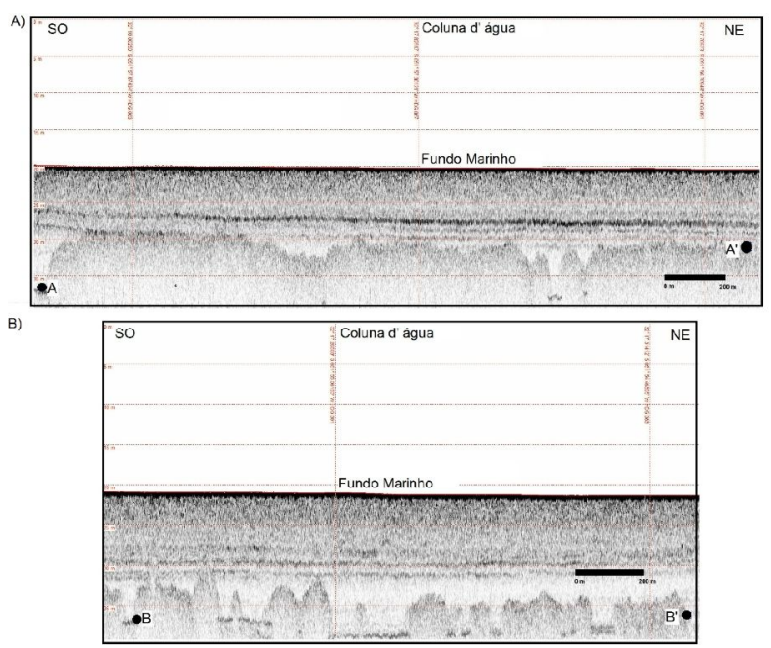

Figura 5 - Perfis sísmicos de gás da linha 4551.

\section{Discussão e Conclusões}

Ocorrências de gás em pacotes sedimentares têm sido detectadas por prospecção sísmica de alta frequência em diversos ambientes costeiros do mundo (GarciaGarcia et al., 1999; Kariiddaiah et al., 1992; Taylor et al., 2000).

A presença de gás nos sedimentos quaternários do substrato da Lagoa dos Patos tem sido evidenciada através do estudo de dados sísmicos de alta resolução (3,5 e 7,0 kHz) (Weschenfelder, 2005; Weschenfelder et al., 2005a; Weschenfelder et al., 2005b; Weschenfelder et al., 2006; Vasconcellos et al., 2006), onde são identificadas feições como bolsões de gás e gás disseminado nos sedimentos.

Dados sísmicos de alta resolução pode ser também uma ferramenta importante para o mapeamento das feições indicativas de acumulação de gás raso. Isto se dá pela anomalia da impedância acústica, pela configuração interna e terminações laterais distintas.

Acumulações de gás ocorrem em áreas com baixos paleotopograficos relacionados à paleocanais fluviais na planície costeira do Rio Grande do Sul. Estas foram 
desenvolvidas durante eventos regressivos / transgressivos do Quaternário (Weschenfelder et al., 2006). As concentrações de gás podem ter se originado a partir da degradação da matéria orgânica depositada nos paleoambientes ali desenvolvidos. Assim, as acumulações de gás estão intimamente relacionadas com os ambientes de sedimentação referenciando os perfis de cortinas de gás registrados neste trabalho.

Os depósitos de fundo da regressão marinha recobertos por sedimentos mais finos e possivelmente menos permeáveis compõem importantes reservatórios de hidrocarbonetos ou água (Zaitlin et al., 1994).

Nos cinco perfis interpretados como gás foram encontradas características como turbidez acústica, reflexões anômalas, perturbações colunares e ocultação de estruturas sedimentares.

Baseado nas características das anomalias acústicas foi possível reconhecer essas "Zonas Acusticamente Turvas" que possuem seu topo bem marcado e terminações laterais abruptas. Estas feições são chamadas de bolsões ou pocket gas, onde se percebe no registro a mudança das camadas sedimentares (meio mais denso) para o gás (meio menos denso). Esse tipo de feição é considerada detentora de maior quantidade de gás, já que camadas plano-paralelas sobrepostas à acumulação atuam como selantes, concentrando o gás produzido sem haver escapes significativos.

\section{Agradecimentos}

Agradecemos ao Programa de Pós Graduação em Oceanografia Física, Química e Geológica e ao Programa de Recursos Humanos da ANP - PRH27 que disponibilizou os recursos necessários para realização deste trabalho.

\section{Referências}

Baptista Neto J. A., Silva M. A. M. \& Figueiredo JR., A.G., 1996. Sísmica de alta frequência e o padrão de distribuição de sedimentos na enseada de Jurujuba (Baía de Guanabara)- RJ/Brasil. Revista Brasileira de Geofísica, 14(1):51-57.

Costa, E. A. \& Figueiredo, A.G., 1998. Echo-character and sedimentary processes on the Amazon Continental Shelf. Anais da Academia Brasileira de Ciências, 70(2):187-200.

Figueiredo A. G., Nittrouer C. A. \& Costa, E. A., 1996. Gas-charged sediments in the Amazon Submarine Delta. Geo-Marine Letters, 16:31-35.

Garcia-Garcia A., Vilas F. \& Garcia-Gil. S. 1999. A seeping sea-floor in Ria environment: Ria de Vigo (Spain). Environment Geology, 38(4):296-300.

Gausland, I. 2003. Seismic surveys impact on fish an fisheries: Repor for Norwegian Oil Industry Association (OLF). Stavanger, 41p.

Karisiddaiah et al. (03 co-authors), 1992. Gas-charged sediments on the inner continental shelf off western India. Marine Geology, 110:143-152.

Manley P. L. \& Flood R. D., 1989. Anomalous sound velocities in near-surface, organic-rich, gassy sediments in the central Argentine basin. Deep-sea Research, 36:611-623.
Neto J. B. O. \& Costa A. J. D., 2007. A Petrobrás e a exploração de Petróleo Offshore no Brasil:um approach Evolucionário, RBE Rio de Janeiro. 61: 95-109p.

Park S. C., Kim Y. S. \& Hong, S. K., 1991. Shallow seismic stratigraphy and distribution pattern of late Quaternary sediments in a macrotidal bay, Gunhung Bay, west coast of Korea. Marine Geology, 98:135-144.

Quaresma V. S., Dias, G. T. M. \& Baptista Neto J. A., 2000. Caracterização da ocorrência de padrões de sonar de varredura lateral e sísmica de alta freqüência (3,5 e 7,0 kHz) na porção sul da Baía de Guanabara RJ. Brazilian Journal of Geophysics, 18(2):201-214.

Taylor M. H., Dillon W. P. \& Pecher I. A., 2000. Trapping and migration of methane associated with the gas hydrate stability zone at the Blake Ridge Diapir: new insights from seismic data. Marine Geology, 164:79-89.

Vasconcellos V. E. B. 2009. Mapeamento e caracterização das acumulações de gás raso na Lagoa dos Patos, sul do Brasil, RS. Porto Alegre, 111p. Dissertação (Mestrado em Geociências), Instituto de Geociências, Universidade Federal do Rio Grande do Sul.

Vasconcellos V. E. B., Weschenfelder J. \& Pereira, C. M. 2006. Acumulações de gás raso no substrato da Lagoa dos Patos revelados por sísmica de alta resolução: caracterização, mapeamento e fatores condicionantes. In: CONGRESSO BRASILEIRO DE GEOLOGIA, 43., 2006. Aracaju SE. Anais..., p. 1.

Vilardo C. 2007. Avaliação ambiental de pesquisas sísmicas marítimas no Brasil : Evolução e Perspectivas. Dissertação de Mestrado, Programa de Planejamento Energético, Universidade Federal do Rio de Janeiro, 307p.

Weschenfelder J., 2005. Processos sedimentares e variação do nível do mar na região costeira do Rio Grande do Sul, Brasil. Porto Alegre, 142p. Tese de Doutorado em Geociências, Instituto de Geociências, Universidade Federal do Rio Grande do Sul.

Weschenfelder J., Corrêa I. C. S. \& Aliotta S., 2005a. Elementos arquiteturais do substrato da Lagoa dos Patos reveladas por sísmica de alta resolução. Pesquisas em Geociências, 32(2): 57-67

Weschenfelder et al. (05 co-authors), 2005b. Gas Accumulations Revealed by High - Resolution Seismic in the Patos Lagoon, Southern Brazil. VIII International Conference on Gas in Marine Sediments. Vigo, Spain;. Cd-rom, In: Geophysics Session.

Weschenfelder et al. (04 co-authors), 2006. Shallow gas accumulation in sediments of the Patos Lagoon, Southern Brazil. Anais da Acadêmia Brasileira de Ciências, 78(3): 607 - 614.

Zaitlin B. A., Dalrymple R. W. \& Boyd R., 1994. The stratigraphic organization of incised valley systems associated with relative sea level change. In: Incised Valley Systems: Origin and Sedimentary Sequences (Dalrymple, R.W., Boyd, R. \& Zaitlin, B.A. eds.), Spec. Publishers Soc. Econ. Paleont. Miner., 51:45 60. 